\title{
Direct Observation of Interfacial Au atoms Using STEM Depth Sectioning
}

\author{
Wenpei Gao ${ }^{1,2}$, Shankar Sivaramakrishnan ${ }^{1,2}$, Jianguo Wen ${ }^{2}$, Jian-Min Zuo ${ }^{1,2}$ \\ ${ }^{1}$ Dept of Materials Science and Engineering, University of Illinois, Urbana-Champaign, IL 61801 \\ ${ }^{2}$ Frederick Seitz Materials Research Laboratory, University of Illinois, Urbana-Champaign, IL \\ 61801
}

Interfacial atoms located between metal nanoparticles and supports are proposed active sites in catalysis, because of their distinct physical and chemical properties [1-3]. However, the atomistic details are difficult to resolve in the interface; the lack of knowledge has been a major obstacle toward unraveling their roles in chemical transformations. Here we report the detection of interfacial $\mathrm{Au}$ atoms on the rutile $\left(\mathrm{TiO}_{2}\right)(110)$ surfaces thanks to the improved spatial resolution and depth of focus brought by aberration corrected scanning transmission electron microscopy (STEM).

$\mathrm{Au}$ on $\mathrm{TiO}_{2}$ is selected because it shows remarkable catalytic activity as the sizes of Au particles reduce to $\sim 3 \mathrm{~nm}$ or below, for the oxidation of $\mathrm{CO}$. The Au catalysts are typically prepared by $\mathrm{Au}$ precipitation on titania support, followed by calcination in air or reduction under $\mathrm{H}_{2}$ at elevated temperatures. Extensive study has been done concerning the mechanism of CO oxidation catalyzed by gold and the role of interfacial gold atoms. However, direct observation of interfacial Au atoms has not been reported before. A major obstacle is the $\mathrm{TiO}_{2}$ support surface, which is often highly complex, undetermined and varies at the nanometer scale. With atomic resolution images recorded at different focuses along $\mathrm{TiO}_{2}$ [001], we have reconstructed the 3D intensity profiles of interfacial atoms. The results lend to direct support to the presence of interfacial Au atoms, embedded in a single interfacial layer.

The experiment started with forming epitaxial Au nanocrystals (NCs) on rutile (110) by e-beam evaporation deposition followed by annealing in air. The sizes of the Au NCs ranged from 3.5 to 12 $\mathrm{nm}$ in width depending upon the annealing conditions. The samples are observed by aberration corrected (AC) scanning transmission electron microscopy (STEM) using the JEOL 2200FS installed at the Center for Microscopy and Microanalysis, Frederick Seitz Materials Research Laboratory, at $200 \mathrm{kV}$. The microscope is capable of resolving atoms separated by $1 \AA$. Figure 1 shows a Z-contrast image recorded along $\mathrm{TiO}_{2}$ [001] direction. Au atoms are brighter then $\mathrm{Ti}$ atoms due to its larger $\mathrm{Z}$. An atomic model of Au nanocrystal on $\mathrm{TiO}_{2}$ [110] surface is shown in the inset, with $\mathrm{Ti}$ atoms in grey, $\mathrm{O}$ atoms in red and $\mathrm{Au}$ in yellow. The arrow indicates a distinct interfacial layer between $\mathrm{Au}$ NCs and the $\mathrm{TiO}_{2}$ (110) surface, with $\mathrm{Au}$ atoms embedded inside the layer.

To locate the interfacial Au atoms in 3D, a focal series of Z-contrast images were recorded first from the Au nanocrystal near the interfacial region shown in Figure 1. The images were aligned using the cross-correlation method. The intensity profiles in the interface layers were then used to form the depth-sectioning images shown in Figure 2, for the $\mathrm{Au}$, $\mathrm{TiO}$ and interfacial layers respectively. The intensity bands were marked using the color boxes to identify the column as a Au-column, Ticolumn or an interfacial-column. Notably, at the interface, the depth-sectioning image shows intermediate intensities between these of $\mathrm{Ti}$ - and Au-columns. Furthermore, there are intensity variations along the focus direction in the interfacial layer. For example, the column marked as $A$ in Figure 2 resembles the intensity of Au columns observed in the Au layer, while the columns marked as $T$ have similar intensities as that of Ti columns in the Ti layer. Regions marked as $I$ show distinct 
intensity peaks that are very different from either the $\mathrm{TiO}$ or $\mathrm{Au}$ layers. Detailed interactions between $\mathrm{Au} \mathrm{NCs}$ and the $\mathrm{TiO}_{2}$ support are thus revealed from these results.

\section{References:}

[1] T. Akita, M. Kohyama, M. Haruta, Accounts of Chemical Research, 46(8), 1773-1782 (2013)

[2] D. Widmann, R.J. Behm, Angewandte Chemie Internatinal Edition, 50(43), 10241 (2011)

[3] Z. Zhou, et al., Advanced Functional Materials, 18(18), 2801-2807 (2008)

[4] The work reported here is supported by the NSF Grant No. DMR 0449790 and AC-STEM was carried out in the Frederick Seitz Materials Research Laboratory Central Facilities, University of Illinois.

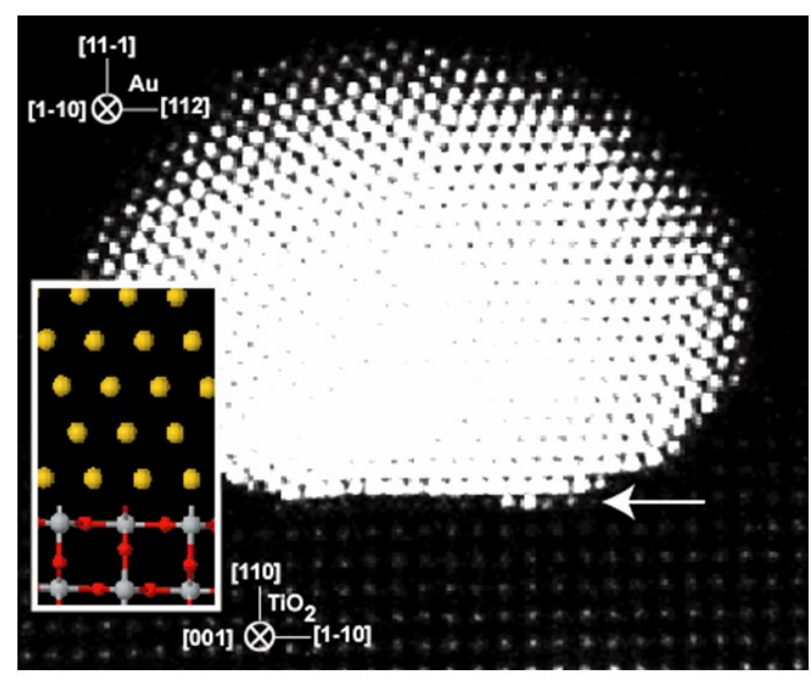

Figure 1

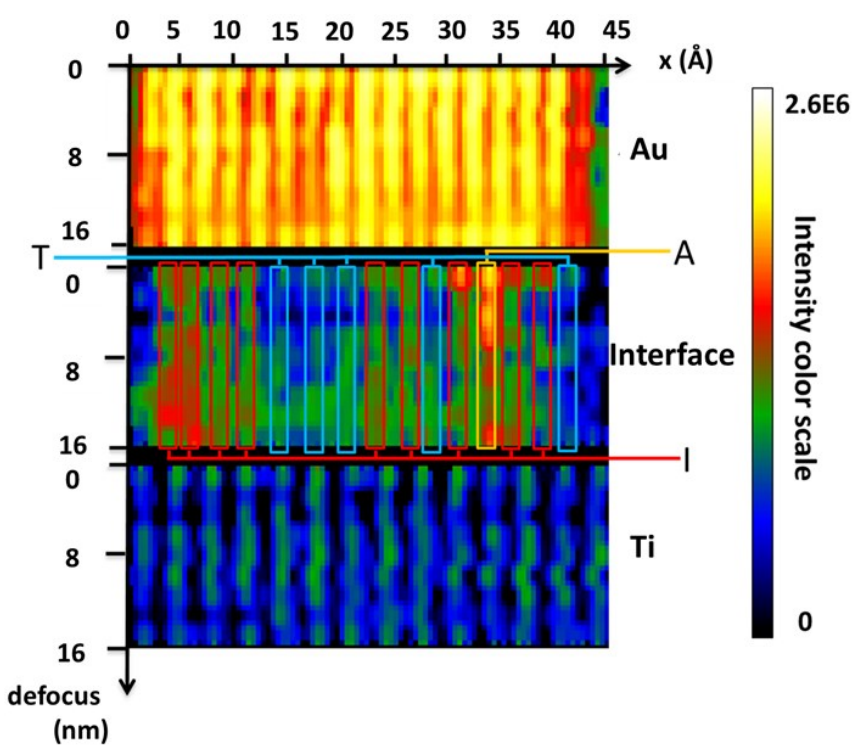

Figure 2

Figure 1, a Z-contrast image recorded along $\mathrm{TiO}_{2}$ [001] direction. Au atoms are brighter then $\mathrm{Ti}$ atoms due to its larger $\mathrm{Z}$. An atomic model of $\mathrm{Au}$ nanocrystal on $\mathrm{TiO}_{2}$ [110] surface is shown in the inset, with $\mathrm{Ti}$ atoms in grey, $\mathrm{O}$ atoms in red and $\mathrm{Au}$ in yellow. A distinct interfacial layer is observed and indicated by the arrow.

Figure 2, the focal series of Z-contrast images recorded from the Au nanocrystal near the interfacial region shown in Figure 1. The images were aligned using the cross-correlation method. These intensity profiles were then used to form the depth-sectioning images shown in Figure 2, for the Au, $\mathrm{TiO}$ and interfacial layers respectively. The intensity bands were marked using the color boxes to identify the column as an Au-column, Ti-column or an interfacial-column. 